\title{
Submillimetre Observations of UC HII Regions and Hot Molecular Cores
}

\author{
G. H. Macdonald and M. A. Thompson \\ Electronics Laboratory, University of Kent, Canterbury, Kent, UK
}

\begin{abstract}
Recent submillimetre observations of continuum radiation from warm dust and molecular line emission from hot gas in regions of high mass star formation are reviewed. Such regions are characterised by ultracompact HII regions around young OB stars and associated hot molecular cores which appear to harbour high mass protostars at an earlier stage of evolution.
\end{abstract}

\section{Introduction}

The aim of this paper is to give a concise overview of the current state of submillimetre observations of ultracompact HII regions (UC HII) and hot molecular cores. By a fortunate coincidence, it is at submillimetre wavelengths that warm dust radiates most strongly in the continuum and hot gas has its greatest density of molecular lines.

UC HII regions are expanding bubbles of highly ionised gas around young early-type $(\mathrm{O}-\mathrm{B} 3)$ stars. They are thus young $\left(<10^{5} \mathrm{yr}\right)$, massive $\left(>10 \mathrm{M}_{\odot}\right)$ and small $(<0.01 \mathrm{pc})$ and the ionized gas is hot $\left(>10^{4} \mathrm{~K}\right)$ and dense $\left(\sim 10^{5}\right.$ $\mathrm{cm}^{-3}$ ). Molecular clouds in which high mass stars are forming are found to be fragmented into clumps of the order of $1 \mathrm{pc}$ in size, often containing denser, hotter cores. These hot molecular cores (HMC, named after the original hot core in Orion-KL) typically have diameter $<0.1 \mathrm{pc}$, density $>10^{7} \mathrm{~cm}^{-3}$ and temperature $>100 \mathrm{~K}$. They are often positionally coincident with $\mathrm{H}_{2} \mathrm{O}, \mathrm{OH}$ and $\mathrm{CH}_{3} \mathrm{OH}$ masers and although usually associated with a UC HII region are normally displaced from it. Hot cores are therefore particularly fascinating because they apparently harbour high mass protostars in proto-OB associations.

\section{UC HII Morphologies and Models}

In their seminal study, Wood \& Churchwell $(1989 \mathrm{a}, \mathrm{b})$ identified over 1600 IRAS point sources that appeared from their infrared colours to be candidate UC HII regions. Subsequent continuum mapping with the VLA of 75 of these objects revealed a variety of morphologies (Figure 1). The largest group were either spherical or unresolved, but the next most common morphology was classified as 'cometary', with a characteristic head and tail structure.

Two competing models have been proposed to explain these cometary structures: 'bow-shock' and 'champagne flow' models. In the bow-shock scenario (Van Buren \& MacLow 1992) the ionising star moves supersonically through 


\section{Ultracompact HII Region Morphologies}
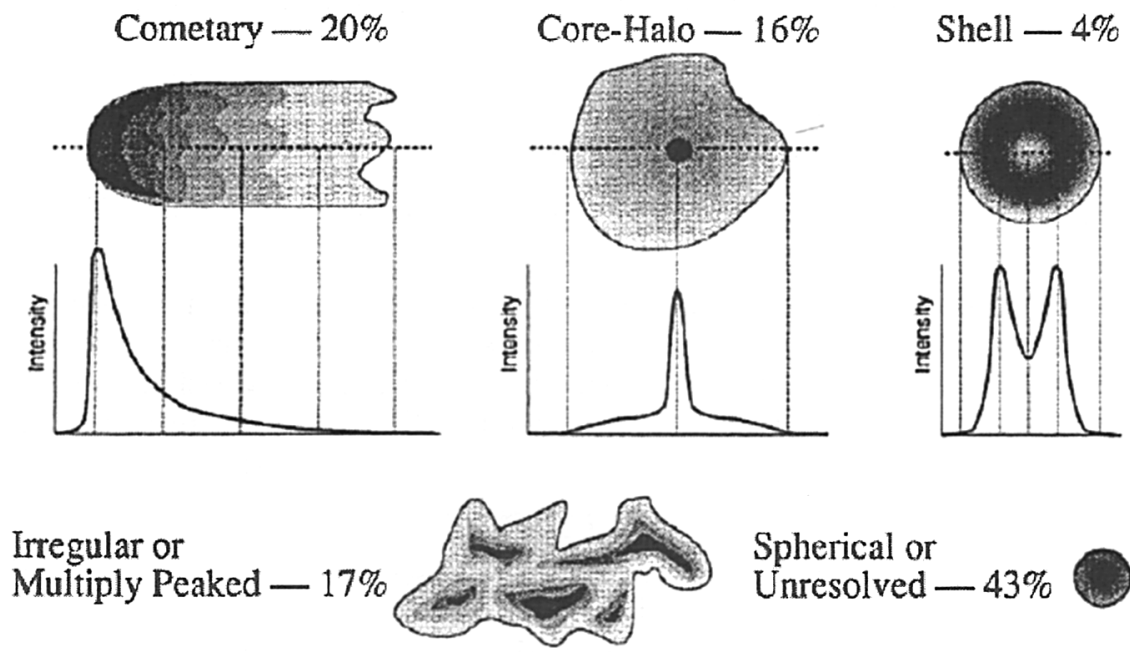

Figure 1. The five morphologies of UC HII regions found by Wood \& Churchwell (1989a).

the ambient medium, piling up shocked gas in a bow shock ahead of it. In the champagne-flow model, the cometary shape arises from the expansion of the HII region into a molecular cloud with a density gradient, with the compact head developing towards the denser gas. Examples can be found where one model provides a better fit to observation in each of these scenarios.

A further difficulty highlighted by Wood \& Churchwell was the so-called 'lifetime problem' where the observed number of UC HII regions appeared to be much greater than expected relative to the numbers of evolved high-mass stars, suggesting their lifetimes were 10-20 times longer than the dynamic lifetime of $\sim 10^{4} \mathrm{yr}$. It is now recognised that Wood \& Churchwell's selection criteria overestimate the number of UC HII regions in the IRAS sample, but only by a factor of around 2-3, so the lifetime problem remains. The lifetime may be extended by providing additional material to soak up ionising photons in the form of photo-evaporating circumstellar disks (Hollenbach et al. 1994) or massloaded stellar winds (Dyson et al. 1995). Alternatively, the expansion can be slowed by a hotter, denser ambient medium (DePree et al. 1995).

However, it seems possible that the lifetime problem may have been exaggerated since recent observations of many UC HII with the VLA in the compact DnC and D configurations by Koo et al. (1996) and Kurtz et al. (1999) have revealed large-scale structures up to $40 \mathrm{pc}$ in size. If UC HII regions are generally embedded in less dense, more extended regions to which earlier surveys were insensitive, then the lifetime problem will need to be reassessed. 

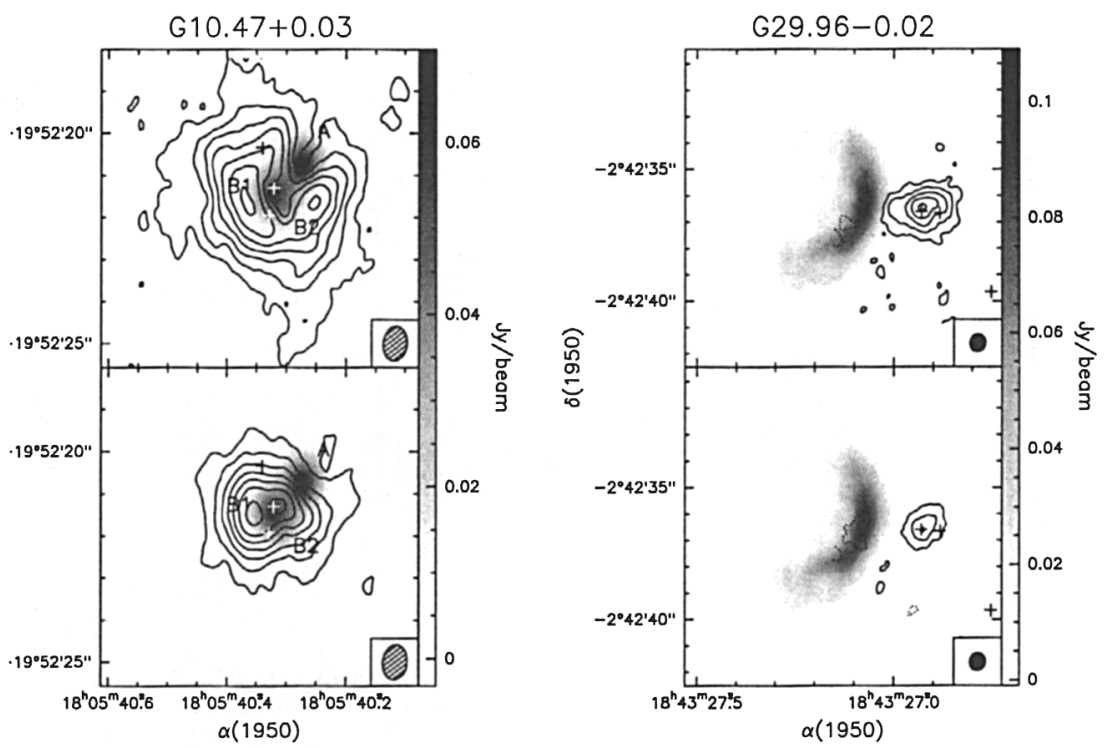

Figure 2. VLA maps at $0.4^{\prime \prime}$ resolution of $\mathrm{NH}_{3}(4,4)$ integrated emission over the main line (top) and all the satellites (bottom), superposed on $1.3 \mathrm{~cm}$ continuum emission (grey scale) from Cesaroni et al. (1998) for G10.47 and G29.92. Crosses indicate $\mathrm{H}_{2} \mathrm{O}$ maser spots (Hofner \& Churchwell 1996).

\section{Examples of UC HII and HMC}

At present, around $175 \mathrm{UC} \mathrm{HII} \mathrm{regions} \mathrm{and} 20$ associated HMC are known (Kurtz et al. 2000). Here we will consider just three interesting examples: G10.47+0.03, G29.96-0.02 and G9.62+0.19, all at similar distances of 6-7 kpc.

Figure 2 shows VLA maps at $0.4^{\prime \prime}$ resolution of excited $\mathrm{NH}_{3}(4,4)$ emission in G10.47 and G29.96, the upper panels showing integrated emission over the main line and the lower panels emission over the optically thin hyperfine lines, superimposed on grey scale maps of the $1.3 \mathrm{~cm}$ continuum emission from the UC HII (Cesaroni et al. 1998). Crosses indicate the positions of $\mathrm{H}_{2} \mathrm{O}$ maser spots (Hofner \& Churchwell 1996).

In G10.47 a cluster of three (A, B1, B2) massive stars, a proto-OB association, is forming which is clearly embedded within the molecular cloud. The kinematics of the gas can be modelled by rotating face-on disks, with blue-shifted $\mathrm{NH}_{3}(4,4)$ absorption interpreted as outflowing gas along the disk axes. G29.96 is a good example of a typical cometary UC HII region where the associated HMC is displaced $\sim 1^{\prime \prime}$ from the vertex, and contains $\mathrm{H}_{2} \mathrm{O}$ maser spots. This whole complex is found to be embedded in a molecular clump $\sim 1 \mathrm{pc}$ in size traced by the $\mathrm{C}^{34} \mathrm{~S}$ (5-4) line (Kurtz et al. 2000). Figure 3 shows a BIMA composite map of $\mathrm{CH}_{3} \mathrm{CN}(6-5)$ and $\mathrm{CH}_{3} \mathrm{OH}$ emission (Pratap et al. 1999), overlaid on a K-band near IR image of G29.96. The $\mathrm{CH}_{3} \mathrm{OH}$ emission is elongated in the direction away from the UC HII region and is displaced $0.2 \mathrm{pc}$ from the peak in $\mathrm{CH}_{3} \mathrm{CN}$, which is surprising since the upper level of the $\mathrm{CH}_{3} \mathrm{OH}$ transition 


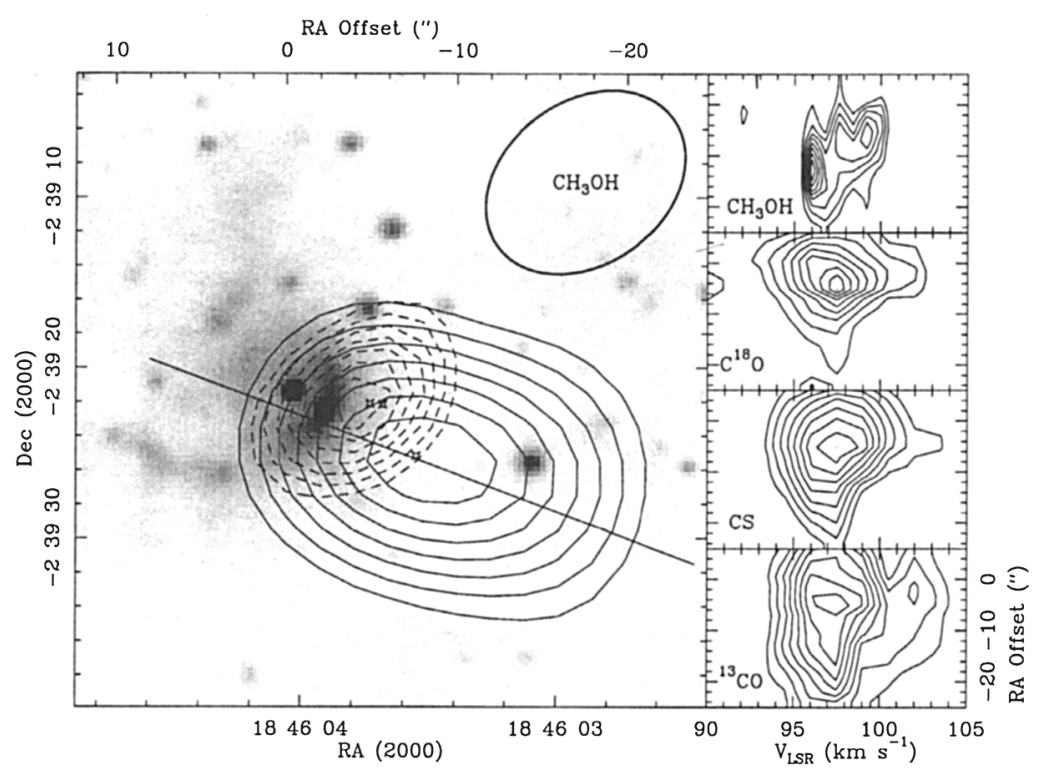

Figure 3. Integrated $\mathrm{CH}_{3} \mathrm{CN}(6-5)$ emission (dashed contours) and $\mathrm{CH}_{3} \mathrm{OH}$ emission (solid contours) overlaid on a K-band image of G29.96-0.02. Inset diagrams are RA-velocity maps. $\mathrm{H}_{2} \mathrm{O}$ masers are shown by star-shaped symbols. Ellipse shows BIMA beam for $\mathrm{CH}_{3} \mathrm{OH}$ (Pratap et al. 1999).

is only $79 \mathrm{~K}$ above ground and might therefore be expected to coincide with the $\mathrm{CH}_{3} \mathrm{CN}$ which has a kinetic temperature of $90 \mathrm{~K}$. The inset RA-velocity diagrams show a pronounced velocity gradient in $\mathrm{CH}_{3} \mathrm{OH}$ which is absent in $\mathrm{CO}$ and CS. Pratap et al. interpret these observations in terms of a molecular outflow enhanced in $\mathrm{CH}_{3} \mathrm{OH}$, possibly arising from a star-forming site indicated by a $\mathrm{H}_{2} \mathrm{O}$ maser at the $\mathrm{CH}_{3} \mathrm{OH}$ peak. These observations indicate a density gradient peaking ahead of the cometary UC HII region and thus favour the champagne flow model, confirming the conclusion of Lumsden \& Hoare (1999) based on the velocity structure of the ionised gas observed in the $2.12 \mu \mathrm{m} \mathrm{1-0} \mathrm{S}(1) \mathrm{H}_{2}$ line. The K-band image shows the exciting star for the cometary nebula and a second star embedded in the UC HII region. Approximately 18 equally reddened stars within $1 \mathrm{pc}^{2}$ of the UC HII are thought to belong to an associated OB cluster.

G9.62 (Figure 4) shows a line of high-mass protostellar objects (C-F) at different stages of evolution, delineated by $\mathrm{NH}_{3}(4,4)$ and $1.3 \mathrm{~cm}$ continuum emission (Cesaroni et al. 1994). F is not detected in centimetric free-free emission, but is seen at $2.2 \mu \mathrm{m}$. Conversely, D is very bright at centimetre wavelengths but is not seen in the near infrared. Testi et al. (1998) interpret these results in terms of an expanding UC HII region. When $\leq 10^{-3} \mathrm{pc}$ in size, the hot dust emits strongly in the near IR but radio emission is self-absorbed (component F). As it expands, the dust cools and it fades in the near IR but becomes detectable in the radio continuum (component $\mathrm{D}$ ). In this model, component $\mathrm{F}$ is the youngest protostellar object in the region, probably a high mass star still in the accreting phase. This conjecture is supported by the presence of a bipolar 


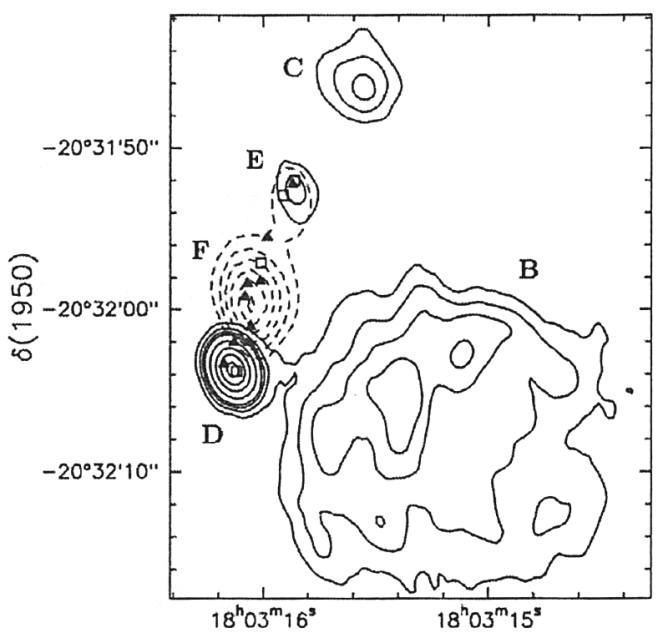

Figure 4. Maps of $\mathrm{NH}_{3}(4,4)$ line (dashed contours) and $1.3 \mathrm{~cm}$ continuum (solid contours) toward G9.62+0.19 (Cesaroni et al. 1994). Filled triangles indicate $\mathrm{H}_{2} \mathrm{O}$ masers (Hofner \& Churchwell 1996) and open squares $\mathrm{OH}$ masers (Forster 1993).

outflow centred on $\mathrm{F}$ (Hofner et al. 1996) and the distribution of $\mathrm{H}_{2} \mathrm{O}$ masers which is concentrated towards this component (Hofner \& Churchwell 1996).

\section{Continuum Observations}

The advent of sensitive bolometric arrays such as SCUBA on JCMT and SHARC on CSO has revolutionised the submm continuum mapping of dust in starforming regions. We have used SCUBA to observe all the UC HII regions mapped by Wood \& Churchwell (1989a) and Kurtz et al. (1994), revealing some very complex structures with multiple peaks, ridges and spurs (Figure 5). Many of the newly discovered peaks are 'radio-quiet', i.e., without embedded UC HII regions, and may be harbouring early massive protostars.

For example, the object at the SE corner of the 2.5' SCUBA field of G34.26+ 0.15 has been found by Hunter et al. (1998) to be a two-component object, a millimetre source G34.24+0.13MM and a near IR source G34.24+0.13IR, separated by $3.4^{\prime \prime}$. The spectral energy distribution (SED) of this composite can be modelled with a $\sim 100 \mathrm{M}_{\odot}$ proto-B star for G34.24+0.13MM, with G34.24+0.13IR an adjacent young star embedded in its own circumstellar envelope. Significantly, a $\mathrm{CH}_{3} \mathrm{OH}$ maser is found in $\mathrm{G} 34.24+0.13 \mathrm{MM}$, thought to be indicative of an earlier evolutionary phase than a $\mathrm{H}_{2} \mathrm{O}$ maser which is associated with a brief phase prior to the formation of a UC HII (Walsh et al. 1998).

The $850 \mu \mathrm{m}$ SCUBA map of G29.96 (Figure 5) shows a N-S ridge of five dust cores, reminiscent of the line of protostellar objects in G9.62. There is 

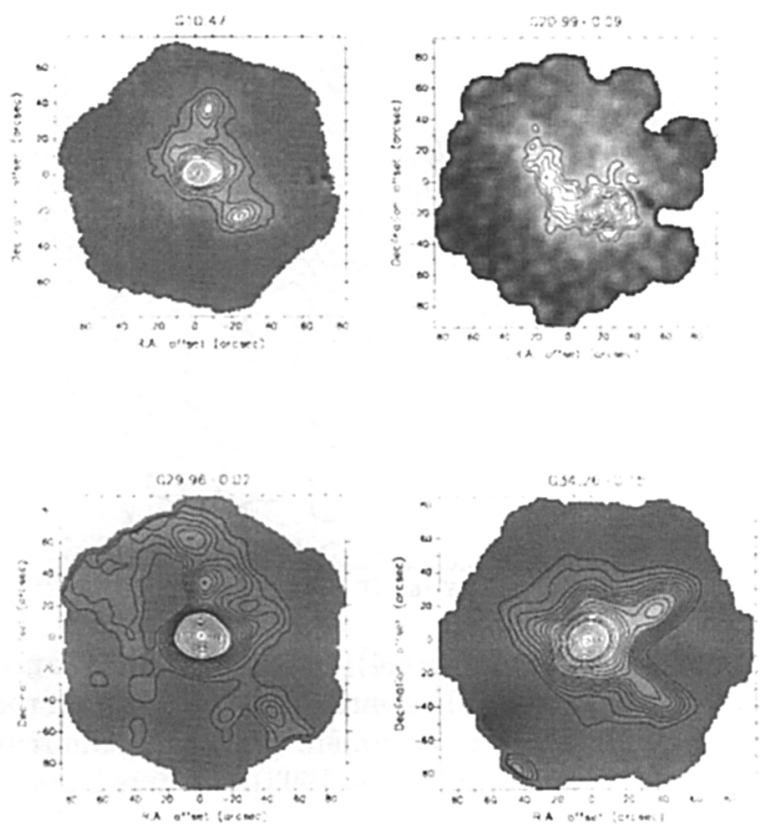

Figure 5. Examples of SCUBA images of UC HII regions at $450 \mu \mathrm{m}$ (G10.47) and $850 \mu \mathrm{m}$ (others), illustrating the complex morphologies of the associated dust.

growing evidence that massive stars tend to form in chains by the collapse and fragmentation of filaments of gas in molecular clouds (Cesaroni et al. 1998).

Further insight into high-mass star formation can be gained by radiative transfer modelling of the SED. Osorio et al. (1999) have found that the SED of four young embedded massive stars (Orion Hot Core, G34.24+0.13MM, W3 $\left(\mathrm{H}_{2} \mathrm{O}\right.$ ) and IRAS $23385+6053$ ) can be modelled by infall of a dusty envelope onto a central OB star with accretion rates of the order of $10^{-3} \mathrm{M}_{\odot} \mathrm{yr}^{-1}$, where the accretion luminosity exceeds the luminosity of the central star. The model can be further constrained by simultaneously fitting to the observed surface brightness distribution.

This approach has also been followed by Hatchell et al. (2000) who used the DUSTY radiative transfer code with a $r^{-3 / 2}$ density profile envelope to model the SED and $850 / 450 \mu \mathrm{m}$ surface brightness profiles of five UC HII regions (G10.47, G12.21, G13.87, G31.41 and G43.89). Of these, two (G13.87 and G43.89) could be modelled without a central high density core (Figure 6), but the remaining three (G10.47, G12.21, G31.41) required the addition of a central object producing up to a third of the total bolometric flux. Significantly, these three hot molecular cores have the richest spectra in this limited sample. 

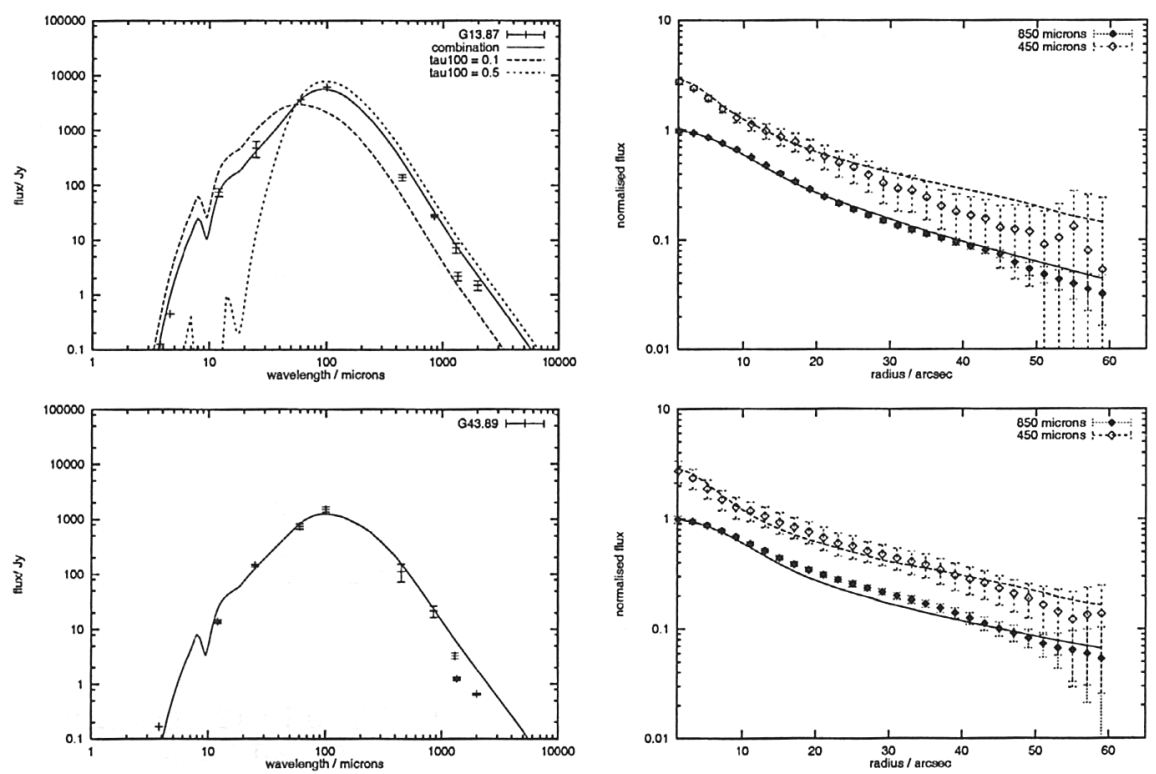

Figure 6. SEDs and radial surface brightness profiles for G13.87 and G43.89 fitted by $r^{-3 / 2}$ density distributions (Hatchell et al. 2000).

\section{Line Observations}

Hot molecular cores display a very rich chemistry comprising a mixture of fully hydrogenated simple species $\left(\mathrm{H}_{2} \mathrm{O}, \mathrm{NH}_{3}, \mathrm{H}_{2} \mathrm{~S}\right)$ and a wide variety of complex organics $\left(\mathrm{CH}_{3} \mathrm{OH}, \mathrm{C}_{2} \mathrm{H}_{5} \mathrm{OH},\left(\mathrm{CH}_{3}\right)_{2} \mathrm{O}, \mathrm{HCOOCH}_{3}, \mathrm{C}_{2} \mathrm{H}_{5} \mathrm{CN} \ldots\right)$ together with high abundances of deuterated species (HDO, DCN, HDCO, $\mathrm{D}_{2} \mathrm{CO}, \mathrm{CH}_{2} \mathrm{DCN}$ ). Chemical timescales can be greater than the lifetime of the UC HII from the switch-on of the exciting star, and so the chemistry retains a 'memory' of the preceding cold accretion phase. At switch-on, simple hydrogenated species which froze out as ice mantles on dust grains are returned to the gas phase, together with more complex species produced by surface chemical processes. The chemistry in HMC therefore provides invaluable information on both the present and past physical conditions during the evolution of high mass stars.

With such a rich chemistry, HMCs have been prime targets for spectral line surveys, both unbiased surveys of individual cores and biased surveys of small samples of cores. At present, such surveys from ground-based telescopes are limited to atmospheric spectral windows until spectrometers on space-borne telescopes such as FIRST become available.

\subsection{Unbiased spectral surveys of individual HMCs}

Unbiased spectral scans have so far been made of relatively few objects, notably Orion (e.g. Schilke et al. 1997), SgrB2 (e.g. Nummelin 1996), W3 $\left(\mathrm{H}_{2} \mathrm{O}\right)$ (Helmich 


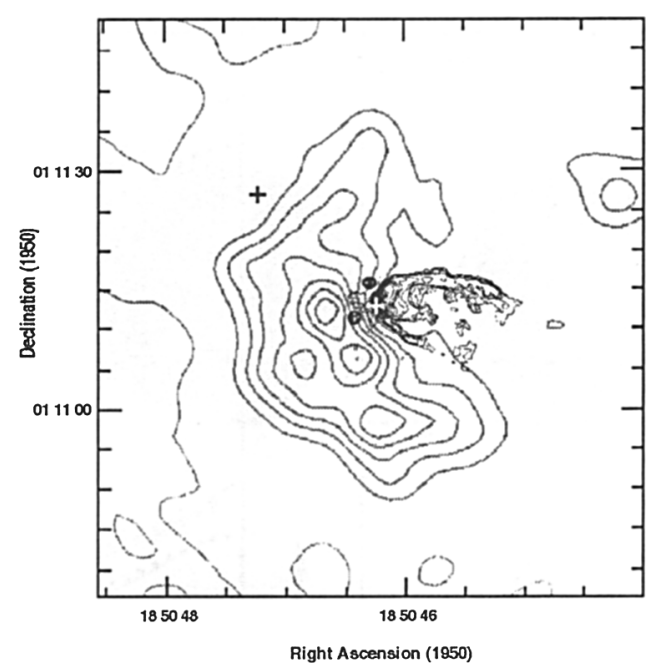

Figure 7. The cometary UC HII region G34.3+0.15 $(2 \mathrm{~cm}$ continuum, shown by black contours; Gaume et al. 1994) and its associated molecular cloud $\left(\mathrm{HCO}^{+}(1-0)\right.$, shown by grey contours; Carral \& Welch 1992). The crosses indicate the spectral survey positions on the HMC (white cross; Macdonald et al. 1996) and the halo (black cross; Thompson et al. 1999).

\& van Dishoeck 1997), G34.3+0.15 (Macdonald et al. 1996) and G5.89-0.39 (Thompson \& Macdonald 1999).

\section{(i) Orion-KL}

The Orion-KL region contains two of the best studied hot cores: the eponymous Orion Hot Core and the compact ridge. Observations of these cores have been extensively reviewed (e.g. Walmsley \& Schilke 1993). In summary, the Orion Hot Core is a few arcseconds in size and peaks at the outflow source IRc2. The fractional abundances of $\mathrm{NH}_{3}, \mathrm{HCN}$ and $\mathrm{H}_{2} \mathrm{~S}$ exceed those for the cooler ambient gas by about one, two and three orders of magnitude respectively, indicative of hydrogenation on grains during the earlier phase of cold accretion. Similarly, the high abundances of $\mathrm{NH}_{2} \mathrm{D}, \mathrm{CH}_{3} \mathrm{OD}$ and $\mathrm{D}_{2} \mathrm{CO}$ arise from deuterium fractionation at very low temperature.

The compact ridge is characterised by enhanced saturated oxygen-bearing molecules such as $\mathrm{CH}_{3} \mathrm{OH}, \mathrm{HCOOCH}_{3}$ and $\left(\mathrm{CH}_{3}\right)_{2} \mathrm{O}$, peaking about $10^{\prime \prime}$ southwest of IRc2. Millar et al. (1991) have shown that the observed abundances of these species can be understood by the early injection of $\mathrm{CH}_{3} \mathrm{OH}$. The high abundance of $\mathrm{NH}_{3}$ in the Orion Hot Core, some 2-3 orders of magnitude higher than the typical value in molecular clouds (Genzel et al. 1982), suggests that the chemical differences between the two hot cores within Orion-KL are simply the result of different thermal history during the period of gravitational collapse (Caselli et al. 1993).

\section{(ii) SgrB2}



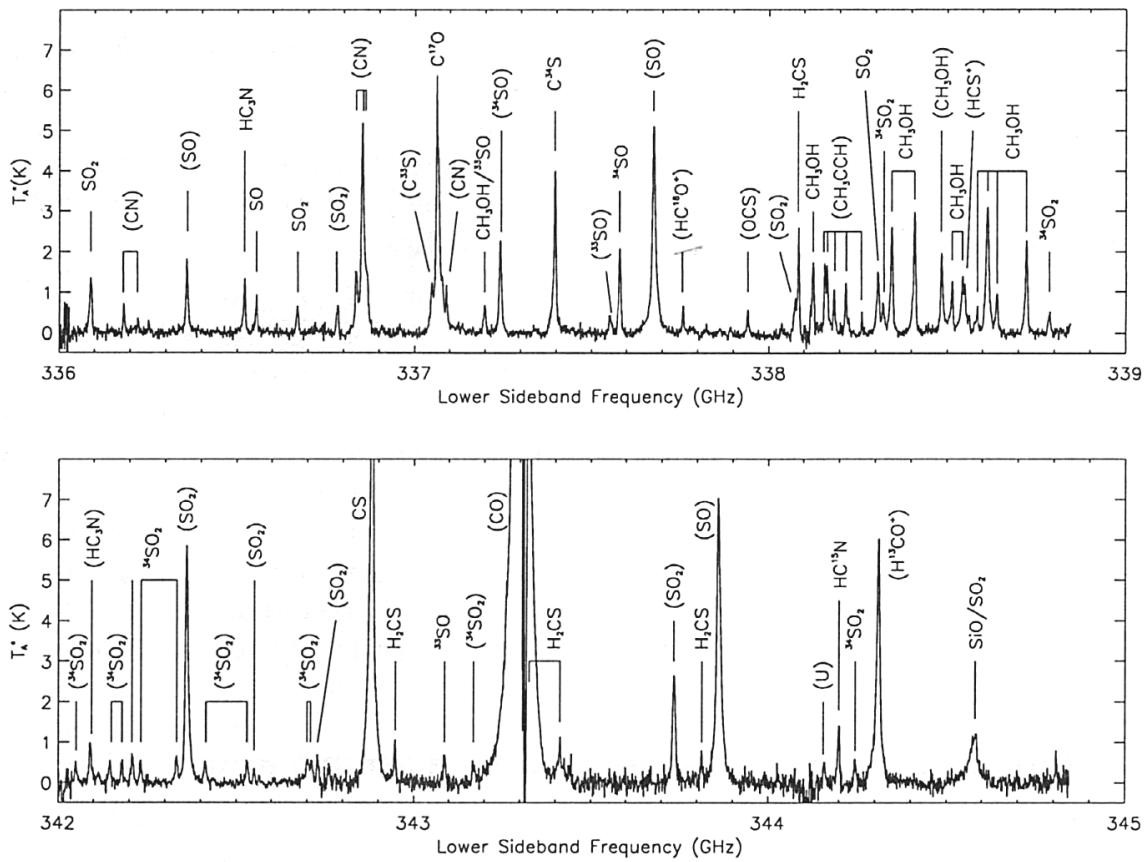

Figure 8. Spectra from the $330-360 \mathrm{GHz}$ survey of the massive outflow source G5.89-0.89 (Thompson \& Macdonald 1999), dominated by sulphur-bearing species such as $\mathrm{SO}$ and $\mathrm{SO}_{2}$.

SgrB2 is a large molecular cloud and HII region complex in the Galactic Centre containing several UC HII regions: SgrB2(OH), SgrB2(M) and SgrB2(N) which have been the subject of several spectral line surveys (e.g. Sutton et al. 1991; Nummelin et al. 1996). However, detailed interpretation of these data is very difficult due to the confusion of structure within a single beam. Resolving this confusion will be a major project for the new generation of submm interferometers.

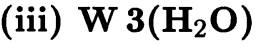

Helmich \& van Dishoeck (1997) carried out an unbiased $345 \mathrm{GHz}$ spectral survey of three sources in the W3 Giant Molecular Cloud: W 3 IRS4, W 3 IRS5 and $\mathrm{W} 3\left(\mathrm{H}_{2} \mathrm{O}\right)$, and found striking chemical differences between them. Silicon and sulphur-bearing molecules such a $\mathrm{SiO}$ and $\mathrm{SO}_{2}$ were prominent towards IRS5 whereas organic molecules such as $\mathrm{CH}_{3} \mathrm{OH},\left(\mathrm{CH}_{3}\right)_{2} \mathrm{O}$ and $\mathrm{CH}_{3} \mathrm{OCHO}$ were more than an order of magnitude more abundant towards W $3\left(\mathrm{H}_{2} \mathrm{O}\right)$. In contrast, only simple molecules were found towards IRS4. They interpret these results in terms of an evolutionary sequence in which IRS5 is the youngest, W $3\left(\mathrm{H}_{2} \mathrm{O}\right)$ somewhat older and IRS4 older still.

\section{(iv) $\mathrm{G34.3}+\mathbf{0 . 1 5}$}

The hot core associated with the UC HII region G34.3+0.15 (Figure 7) appears to be a single object and so is much easier to study than the hot cores in Orion-KL and SgrB2. Macdonald et al. (1996) performed a $330-360 \mathrm{GHz}$ scan of this HMC and found an exceptionally rich chemistry with more than 35 
distinct species. The measured abundances have been modelled by Millar et al. (1997) in a chemical evolutionary model which, for the first time, included the structure of the HMC with three distinct components: an ultracompact core, a compact core and an extended halo. The column densities in the halo region were subsequently measured (Thompson et al. 1999) and the model extended to yield more realistic beam-averaged column densities.

\section{(v) G5.89-0.39}

Molecular gas associated with the UC HII region G5.89-0.39 shows evidence of a massive outflow (Harvey \& Forveille 1988; Acord et al. 1997). Although low-mass YSO outflows are common, until recently very few examples in high-mass YSOs were known. However, recent studies by Shepherd \& Churchwell $(1996 \mathrm{a}, \mathrm{b})$ have shown high-velocity CO emission to be present in $90 \%$ of massive YSOs investigated. An unbiased 330-360 GHz line survey by Thompson \& Macdonald (1999) detected 19 species with more than half being sulphurbearing and with no emission from heavy organic molecules (Figure 8). The very different chemistry seen in this HMC almost certainly results from shocks due to the outflow.

\subsection{Biased spectral surveys of a hot core sample}

A complementary approach to studying the chemistry in HMCs is to make limited spectral surveys including lines from selected species for a large number of molecular cores. Biased surveys of this type toward 14 UC HII regions detected wide variations in chemical complexity and excitation (Hatchell et al. 1998a) which could be divided almost equally into 'line-rich' and 'line-poor' categories. The line-rich sources showed clear evidence for a core-halo structure, with a hot $(>80 \mathrm{~K})$, dense $\left(>10^{8} \mathrm{~cm}^{-3}\right)$, compact $(<0.05 \mathrm{pc})$ core surrounded by cooler ambient gas. The line-poor sources could be modelled without a hot core by a $20-30 \mathrm{~K}, 10^{5} \mathrm{~cm}^{-3}$ cloud. No correlation could be found between UC HII region size or shape and core size, chemical evolution, ambient cloud density or linewidth. The column densities of most species except $\mathrm{CH}_{3} \mathrm{CN}$ and $\mathrm{HCOOCH}_{3}$ could be modelled satisfactorily. A detailed study of the sulphur chemistry in the sample indicated that they were all at a similar evolutionary stage (Hatchell et al. 1998b).

\section{Conclusions}

Submillimetre observations, both spatial and spectral, of UC HII regions and hot molecular cores have made rapid advances in the past few years, due mainly to the appearance of highly sensitive bolometer arrays. Wide-field mapping of the associated dust reveals many 'radio-quiet' clumps which may harbour high mass stars before the formation of a UC HII region. Biased and unbiased spectral surveys of hot cores are yielding valuable information on their chemical evolution which, through careful chemical modelling, potentially can give useful insight into the cold accretion phase of massive star formation. Interferometry in both line and continuum is revealing the detailed distributions of gas and dust in such regions but the very high submm resolution of instruments such as ALMA is required to unravel the detailed relation between UC HII regions and hot molecular cores. Finally, there is a pressing need to identify a larger 
sample of high-mass protostars for chemical and statistical studies and for this their association with methanol masers will be valuable means of identification.

\section{References}

Acord, J.M., Walmsley, C.M., \& Churchwell, E. 1997, ApJ, 475, 693

Carral, P. \& Welch, W.J. 1992, ApJ, 385, 244

Caselli, P., Hasegawa, T.J., \& Herbst, E. 1993, ApJ, 405, 548

Cesaroni, R., Churchwell, E., Hofner, P., Walmsley, C.M., \& Kurtz, S. 1994, A\&A, 288, 903

Cesaroni, R., Hofner, P., Walmsley, C.M., \& Churchwell, E. 1998, A\&A, 331, 709

DePree, C.G., Rodriguez, L.F., \& Goss, W.M. 1995, Rev. Mexicana Astron. Astrofis., 31, 39

Dyson, J.E., Williams, R.J.R., \& Redman, M.P. 1995, MNRAS, 277, 700

Forster, J.R. 1993, Astrophysical Masers, eds. A.W. Clegg \& G.E. Nedoluha (Berlin: Springer-Verlag)

Gaume, R.A., Fey, A.L., \& Claussen, M.J. 1994, ApJ, 432, 638

Genzel, R., Downes, D., Ho, P.T.P., \& Bieging, J.H. 1982, ApJ, 259, L103

Harvey, P.M. \& Forveille, T. 1988, A\&A, 197, L19

Hatchell, J., Fuller, G.A., Millar, T.J., \& Thompson, M.A., Macdonald, G.H. 2000, A\&A, in press

Hatchell, J., Thompson, M.A., Millar, T.J., \& Macdonald, G.H. 1998a, A\&AS, 133,29

1998b, A\&A, 338, 713

Helmich, F.P. \& van Dishoeck, E.F. 1997, A\&AS, 124, 205

Hofner, P. \& Churchwell, E. 1996, A\&AS, 120, 283

Hofner, P., Kurtz, S., Churchwell, E., Walmsley, C.M., \& Cesaroni, R. 1996, ApJ, 460, 359

Hollenbach, D., Johnstone, D., Lizano, S., \& Shu, F. 1994, ApJ, 428, 654

Hunter, T.R., Neugebauer, G., Benford, D.J., Matthews, K., Lis, D.C., Serabyn, E., \& Phillips, T.G. 1998, ApJ, 493, L97

Koo, B.-C., Kim, K.-T., Lee, H.-G., Yun, M.-S., \& Ho, P.T. 1996, ApJ, 456, 662

Kurtz, S.E., Cesaroni, R., Churchwell, E., Hofner, P., \& Walmsley, C.M. 2000, Protostars and Planets IV, Univ. Arizona Press

Kurtz, S.E., Churchwell, E., \& Wood, D.O.S. 1994, ApJS, 91, 659

Kurtz, S.E., Watson, A.M., Hofner, P., \& Otte, B. 1999, ApJ, 514, 232

Lumsden, S.L. \& Hoare, M.G. 1999, MNRAS, 305, 701

Macdonald, G.H., Gibb, A.G., Habing, R.J., \& Millar, T.J. 1996, A\&AS, 119, 333

Millar, T.J., Herbst, E., \& Charnley, S.B. 1991, ApJ, 369, 147

Millar, T.J., Macdonald, G.H., \& Gibb, A.G. 1997, A\&A, 325, 1163

Nummelin, A. 1996, Tech. Report 256L (Göteborg: Chalmers Univ)

Osorio, M., Lizaro, S., \& D'Alessio, P. 1999, ApJ, 525, 808

Pratap, P., Megeath, S.T., \& Bergin, E.A. 1999, ApJ, 517, 799

Schilke, P., Groesbeck, T.D., Blake, G.A., \& Phillips, T.G. 1997, ApJS, 108, 301

Shepherd, D.S. \& Churchwell, E. 1996a, ApJ, 457, 267

1996b, ApJ, 472, 225

Sutton, E.C., Jaminet, P.A., Danchi, W.C., \& Blake, G.A. 1991, ApJS, 311, 921

Testi, L., Felli, M., Persi, P., \& Roth, M. 1998, A\&A, 329, 233 
Thompson, M.A. \& Macdonald, G.H. 1999, A \& AS, 135, 531

Thompson, M.A., Macdonald, G.H., \& Millar, T.J. 1999, A \& A, 342, 809

Van Buren, D., MacLow, M.-M. 1992, ApJ, 394, 534

Walmsley, C.M. \& Schilke, P. 1993, in Dust and Chemistry in Astronomy, eds. Millar, T.J. \& Williams, D.A., IOP Publishing, Bristol

Walsh, A.J., Burton, M.G., Hyland, A.R., \& Robinson, G. 1998, MNRAS, 301, 640

Wood, D.O.S. \& Churchwell, E. 1989a, ApJS, 69, 831 1989b, ApJ, 340, 265

\section{Discussion}

J. Rawlings: Even with the higher spatial resolutions offered by high frequency observations, the beam size of single dish telescopes (several arcsecs) samples regions of very different physical characteristics. Other than some (albeit important) general qualitative results would you agree that it is difficult to obtain quantitative information from the spectral line surveys?

G. Macdonald: Yes, it has to be born in mind that all single-dish spectral observations give a beam-averaged spectrum over all the structures within the beam. A good example would be the Orion Hot Core and Compact Ridge regions. Another problem is the high optical depth sometimes seen in some lines which must be corrected for in some way for derived physical parameters to be reliable. In general, measured abundances may often only be correct to an order of magnitude, but even so can be useful.

M. Burton: While the timescale problem for the lifetime of UC HII regions may now be alleviated, or no longer a problem, has it not moved to an earlier stage of star formation - the hot core and/or protostellar condensation stage? Surveys for UC HII regions based on Wood \& Churchwell colors are finding large numbers without radio emission, but nevertheless have all the signs that massive star formation is taking place (masers, mid-IR sources, outflows, etc.). Yet their lifetime can also be no longer than $\sim 10^{4}$ years?

G. Macdonald: If hot cores are fragments of a molecular cloud remaining after the formation of high-mass stars, their numbers must be arbitrary. However, if hot cores eventually spawn further massive stars there could be a lifetime problem if the hot core phase lasts for less than $10^{5}$ years, assuming that only one star forms in each core, or even shorter than this time if more do. 